九州大学学術情報リポジトリ

Kyushu University Institutional Repository

\title{
A New Lignan Having A Chromene Moiety: Synthesis of Haedoxan Analogue with Precocene I Skeleton
}

Hirata, Makoto

Laboratory of Pesticide Chemistry, Faculty of Agriculture, Kyushu University

Taniguchi, Eiji

Laboratory of Pesticide Chemistry, Faculty of Agriculture, Kyushu University

https://doi.org/10.5109/24136

出版情報：九州大学大学院農学研究院紀要. 41 (1/2)，pp.113-124，1996-11. Kyushu University バージョン：

権利関係 : 


\title{
A New Lignan Having A Chromene Moiety: Synthesis of Haedoxan Analogue with Precocene I Skeleton
}

\section{Makoto Hirata and Eiji Taniguchi*}

\author{
Laboratory of Pesticide Chemistry, Faculty of Agriculture, \\ Kyushu University, Fukuoka 812-81, Japan \\ (Received July 31, 1996)
}

\begin{abstract}
A new lignoid replacing the 6-methoxy-2-methoxymethyl-(3,4-methylenedioxyphenyl)-1,4benzodioxan-7-yl group of an insecticidal lignan, haedoxan, to an anti-juvenile hormone, 2,2dimethyl-7-methoxychromene (precocene I), was synthesized from 3-benzyloxy-2,2-dimethyl-7methoxychroman-6-carbaldehyde through 16 steps in a $0.92 \%$ overall yield. This analogue showed no insect growth-regulatory activity at a level of 200pg dose in a bioassay using the 4th instar larvae of the silkworm.
\end{abstract}

\section{INTRODUCTION}

Haedoxan A, (+ )- $(1 S, 2 R, 5 R, 6 S, 2 " R, 3 " R)-1$-hydroxy-2-(2',6'-dimethoxy-3',4'methylenedioxyphenoxy)-6-[2",3"-dihydro-6"-methoxy-2"-methoxymethyl-3"-(3,4-methylenedioxyphenyl)-1",4"-benzodioxine-7"-yl]-3,7-dioxabicyclo[3.3.0]octane, was isolated as the main constituent of the insecticidal neolignans of Phryma leptostachya L. (haedokusou) (Taniguchi et al., 1989). The 1,4-benzodioxan framework in this unique sesquilignan was essential for its insecticidal activity (Yamauchi and Taniguchi, 1991, $1992 \mathrm{a}, \mathrm{b}, \mathrm{c}, \mathrm{d})$. In a challenge to explore new biological activities, a haedoxan analogue (21) possessing antiJH hormone (precocene I isolated from Ageratum Xoustonianum) (Bowers and Ohta, 1976) structure resembling to 1,4-benzodioxan framework of haedoxan was synthesized (Fig. 1), and assayed on the 4th instar larvae of the silkworm.

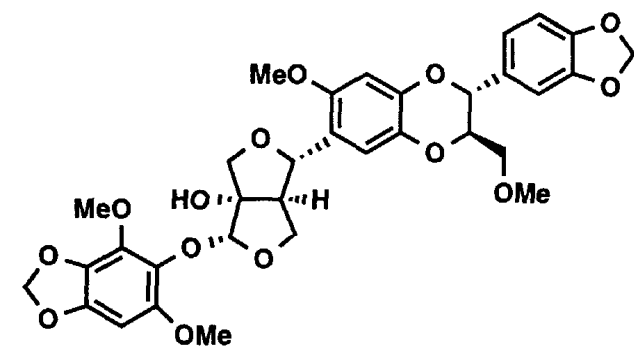

(+)-Haedoxan A

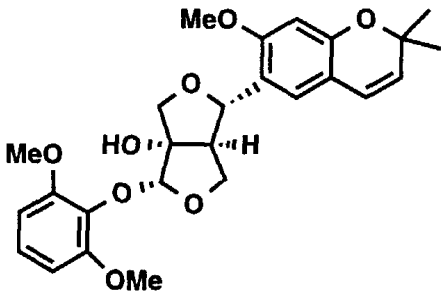

21

Fig. 1. The structures of(+)-Haedoxan A and analogue (21).

\footnotetext{
* To whom correspondence should be addressed.
} 


\section{MATERIALS AND METHODS}

All melting points (mp.) and boiling point (bp.) are uncorrected. 'H-NMR spectra were measured with a JEOL-EX 400 spectrometer, and chemical shifts are reported as values in parts per million relative to tetramethylsilane $(\delta$ н 0.0$)$ as an internal standard. Data are reported as the chemical shifts (integrated intensity, multiplicity and coupling constant). Gravity column chromatography was carried out with Merck silica-gel 60 (230400 mesh ASTM). Preparative TLC was performed on precoated $60 \mathrm{~F}_{254}$ silica-gel plates supplied by E. Merck.

2,2-Dimethyl-7-methoxychromene (precocene I) (1) . Precocene I was prepared from 3-methoxyphenol $(11.0 \mathrm{ml}, 0.10 \mathrm{~mol})$ and 3,3-dimethylacrylic acid $(11.0 \mathrm{~g}, 0.11 \mathrm{~mol})$ according to the method described in the literature (Ohta and Bowers, 1977). A colourless oil $(11.8 \mathrm{~g}, 62 \%)$. bp. $125-128{ }^{\circ} \mathrm{C} / 4 \mathrm{mmHg}$ (lit. bp. $97-100{ }^{\circ} \mathrm{C} / 2 \mathrm{mmHg}$ ). NMR $\delta \mathrm{H}$ $\left(\mathrm{CDCl}_{3}\right): 1.42(6 \mathrm{H}, \mathrm{S}), 3.77(3 \mathrm{H}, \mathrm{S}), 5.47(1 \mathrm{H}, \mathrm{d}, J=9.77 \mathrm{~Hz}), 6.27(1 \mathrm{H}, \mathrm{d}, J=9.77 \mathrm{~Hz}), 6.37$ $(1 \mathrm{H}, \mathrm{d}, J=2.44 \mathrm{~Hz}), 6.88(1 \mathrm{H}, \mathrm{d}, J=2.44,8.30 \mathrm{~Hz}), 7.74(1 \mathrm{H}, \mathrm{d}, J=8.30 \mathrm{~Hz})$. Anal. Found: C, 75.67; H, 7.51. Calcd. for $\mathrm{C}_{12} \mathrm{H}_{14} \mathrm{O}_{2}: \mathrm{C}, 75.75 ; \mathrm{H}, 7.42 \%$.

2,2-Dimethyl-7-methoxychroman-3,4-diol (2). A mixture of precocene I (10.0 g, 52.6 mmol), $N$-methylmorpholine $\mathrm{N}$-oxide (NMO; $6.80 \mathrm{~g}, 57.9 \mathrm{mmol}$ ) and $\mathrm{OsO}_{4}(2 \mathrm{ml}$ of a $2 \%$ aqueous solution) in acetone $(140 \mathrm{ml})$, tert-BuOH $(70 \mathrm{ml})$ and $\mathrm{H}_{2} \mathrm{O}(70 \mathrm{ml})$ was stirred at room temperature for $72 \mathrm{hr}$ under $\mathrm{N}_{2}$ atmosphere in the dark. After quenching (aqueous soln. of $\mathrm{NaHSO}_{3}$ ), the reaction mixture was filtered through a celite pad, and the filtrate was evaporated. The residue was extracted with EtOAc. The extract was dried over $\mathrm{Na}_{2} \mathrm{SO}_{4}$, filtered, and the solvent was evaporated. The residue was purified by silica-gel chromatography (eluent; hexane $: E t O A c=2: 1)$ to obtain $2(7.0 \mathrm{~g}, 58 \%)$ as white crystals. mp. 102-103 "C. NMR $\delta \mathrm{H}\left(\mathrm{CDCl}_{3}\right): 1.30(3 \mathrm{H}, \mathrm{S}), 2.01(1 \mathrm{H}, \mathrm{d}, J=8.30 \mathrm{~Hz}), 2.47(1 \mathrm{H}, \mathrm{d}$, $J=9.28 \mathrm{~Hz}), 3.70(1 \mathrm{H}, \mathrm{dd}, J=4.40,8.30 \mathrm{~Hz}), 3.77(3 \mathrm{H}, \mathrm{S}), 4.77(1 \mathrm{H}, \mathrm{dd}, J=4.40,9.28 \mathrm{~Hz})$, $6.37(1 \mathrm{H}, \mathrm{d}, J=2.44 \mathrm{~Hz}), 6.58(1 \mathrm{H}, \mathrm{dd}, J=2.44,7.81 \mathrm{~Hz}), 7.41(1 \mathrm{H}, \mathrm{d}, J=7.81 \mathrm{~Hz})$. Anal. Found: C, 64.21; $\mathrm{H}, 7.12$. Calcd. for $\mathrm{C}_{12} \mathrm{H}_{16} \mathrm{O}_{4}: \mathrm{C}, 64.26 ; \mathrm{H}, 7.20 \%$.

2,2-Dimethyl-7-methoxychroman-3-one (3). To a stirred solution of 2 (7.0 g, 30.7 mmol) in THF $(200 \mathrm{ml})$ was added conc. $\mathrm{HCl}(10 \mathrm{ml})$ and refluxed for $\mathrm{hr}$. The solution was neutralized with sat. $\mathrm{NaHCO}_{3}$ solution and extracted by EtOAc. The extract was washed with brine, dried over $\mathrm{Na}_{2} \mathrm{SO}_{4}$, filtered and concentrated. The crude product was purified by silica-gel chromatography (eluent; hexane : EtOAc $=4: 1)$ to obtain $3(6.23 \mathrm{~g}$, quant.) as a colourless syrup. NMR $\delta \mathrm{H}\left(\mathrm{CDCl}_{3}\right): 1.41(6 \mathrm{H}, \mathrm{S}), 3.53(2 \mathrm{H}, \mathrm{S}), 3.79(3 \mathrm{H}, \mathrm{S})$, 6.53-6.61 (2H, m), $6.98(1 \mathrm{H}, \mathrm{d}, J=8.30 \mathrm{~Hz})$.

2,2-Dimethyl-7-methoxychroman-3-ol (4). To a stirred suspension of $\mathrm{LiAlH}_{4}(0.76 \mathrm{~g}$, $22.4 \mathrm{mmol})$ in dry THF $(100 \mathrm{ml})$ was added a solution of $3(6.0 \mathrm{~g}, 29.1 \mathrm{mmol})$ in dry THF $(100 \mathrm{ml})$ at room temperature and stirred for $2 \mathrm{hr}$. The reaction mixture was quenched by the addition of sat. $\mathrm{MgSO}_{4}$ soln. and anhydrous $\mathrm{K}_{2} \mathrm{CO}_{3}$, and stirred for $2 \mathrm{hr}$. The mixture was filtered through a celite pad, and the filtrate was concentrated. The crude product was purified by silica-gel chromatography (eluent; hexane :EtOAc $=4: 1)$ to obtain 4 $(4.88 \mathrm{~g}, 81 \%)$ as colourless crystals. mp. $65-66{ }^{\circ} \mathrm{C} . \mathrm{NMR} \delta \mathrm{H}\left(\mathrm{CDCl}_{3}\right): 1.32(3 \mathrm{H}, \mathrm{S}), 1.36$ $(3 \mathrm{H}, \mathrm{S}), 1.72(1 \mathrm{H}, \mathrm{d}, J=7.81 \mathrm{~Hz}), 2.71(1 \mathrm{H}, \mathrm{dd}, J=5.37,16.6 \mathrm{~Hz}), 3.01(1 \mathrm{H}, \mathrm{dd}, J=4.89$, $16.6 \mathrm{~Hz}), 3.76(3 \mathrm{H}, \mathrm{S}), 3.77(1 \mathrm{H}, \mathrm{m}), 6.40(1 \mathrm{H}, \mathrm{d}, J=2.44 \mathrm{~Hz}), 6.48(1 \mathrm{H}, \mathrm{dd}, J=2.44,8.30$ $\mathrm{Hz}), 6.92(1 \mathrm{H}, \mathrm{d}, J=8.30 \mathrm{~Hz})$. Anal. Found: C, 69.22; H, 7.73. Calcd. for $\mathrm{C}_{12} \mathrm{H}_{16} \mathrm{O}_{3}: \mathrm{C}, 69.19$; 


\section{H. $7.75 \%$.}

3-Benzyloxy-2,2-dimethyl-7-methoxychroman (5). To a stirred and ice-cooled suspension of $\mathrm{NaH}(0.92 \mathrm{~g}$, a $60 \%$ dispersion in mineral oil, $23.0 \mathrm{mmol})$ in dry THF (100 ml) was added a solution of $4(4.73 \mathrm{~g}, 22.74 \mathrm{mmol})$ in dry THF $(50 \mathrm{ml})$ under $\mathrm{N}_{2}$ atmosphere. The mixture was allowed to reach room temperature and stirred for $30 \mathrm{~min}$. To the mixture was added benzyl bromide $(4.1 \mathrm{ml}, 34.5 \mathrm{mmol})$ and ${ }^{\mathrm{n}} \mathrm{Bu} \mathrm{u}_{4} \mathrm{NI}(0.2 \mathrm{~g})$, and stirred at room temperature for $20 \mathrm{hr}$ before quenching by a addition of $\mathrm{H}_{2} \mathrm{O}(30 \mathrm{ml})$. The reaction mixture was extracted by EtOAc. The extract was washed with brine, dried over $\mathrm{Na}_{2} \mathrm{SO}_{4}$, filtered and concentrated. The residue was purified by silica-gel chromatography (eluent; hexane : EtOAc $=20: 1)$ to obtain $5(5.94 \mathrm{~g}, 88 \%)$ as a colourless oil. NMR $\delta \mathrm{H}\left(\mathrm{CDCl}_{3}\right)$ : $1.27(3 \mathrm{H}, \mathrm{S}), 1.39(3 \mathrm{H}, \mathrm{S}), 2.57(1 \mathrm{H}, \mathrm{dd}, J=8.30,15.62 \mathrm{~Hz}), 2.96(1 \mathrm{H}, \mathrm{dd}, J=5.37,15.62$ $\mathrm{Hz}), 3.54(1 \mathrm{H}, \mathrm{dd}, J=5.37,8.30 \mathrm{~Hz}), 3.74(3 \mathrm{H}, \mathrm{S}), 4.55(1 \mathrm{H}, \mathrm{d}, J=12.21 \mathrm{~Hz}), 4.72(1 \mathrm{H}, \mathrm{d}$, $J=12.21 \mathrm{~Hz}), 6.37(1 \mathrm{H}, \mathrm{d}, J=2.41 \mathrm{~Hz}), 6.45(1 \mathrm{H}, \mathrm{m}), 6.93(1 \mathrm{H}, \mathrm{d}, J=8.30 \mathrm{~Hz}), 7.29(1 \mathrm{H}$, m), $7.34(2 \mathrm{H}, \mathrm{S}), 7.35(2 \mathrm{H}, \mathrm{S})$. Anal. Found: C, 76.47; H, 7.43. Calcd. for $\mathrm{C}_{19} \mathrm{H}_{22} \mathrm{O}_{3}: \mathrm{C}$, 76.47; H, 7.44\%.

3-Benzyloxy-2,2-dimethyl-7-methoxychroman-6-carbaldehyde (6). To a cooled (0 “C) solution of $5(1.42 \mathrm{~g}, 4.76 \mathrm{mmol})$ in dry DMF $(10 \mathrm{ml})$ was added $\mathrm{POCl}_{3}(0.82 \mathrm{ml}, 9.52$ mmol) as drops. The mixture was stirred at room temperature for $30 \mathrm{~mm}$., then stirred at $80{ }^{\circ} \mathrm{C}$ for $3 \mathrm{hr}$. After cooling to room temperature, the solution was quenched by $\mathrm{NaOAc}$ solution ( $4 \mathrm{~g}$ in $18 \mathrm{ml} \mathrm{H}_{2} \mathrm{O}$ ). The mixture was extracted by $\mathrm{Et}_{2} \mathrm{O}$. The extract was washed with sat. $\mathrm{NaHCO}_{3}$, brine, dried over $\mathrm{Na}_{2} \mathrm{SO}_{4}$, filtered and concentrated. The residue was purified by silica-gel chromatography (eluent; hexane : EtOAc= $8: 1)$ to obtain $6(1.37 \mathrm{~g}$, $88 \%)$ as a colourless oil. NMR $\delta \mathrm{H}\left(\mathrm{CDCl}_{3}\right): 1.33(3 \mathrm{H}, \mathrm{S}), 1.38(3 \mathrm{H}, \mathrm{S}), 2.78(1 \mathrm{H}$, dd, $J=7.32,16.11 \mathrm{~Hz}), 2.96(1 \mathrm{H}, \mathrm{dd}, J=4.89,16.11 \mathrm{~Hz}), 3.54(1 \mathrm{H}, \mathrm{dd}, J=4.88,7.32 \mathrm{~Hz}), 3.85$ $(3 \mathrm{H}, \mathrm{S}), 4.53(1 \mathrm{H}, \mathrm{d}, J=12.21 \mathrm{~Hz}), 4.71(1 \mathrm{H}, \mathrm{d}, J=12.21 \mathrm{~Hz}), 6.38(1 \mathrm{H}, \mathrm{S}), 7.28-7.36(5 \mathrm{H}$, m) , $7.58(1 \mathrm{H}, \mathrm{S}), 10.26(1 \mathrm{H}, \mathrm{S})$. Anal. Found: C, 73.49; H, 6.88. Calcd. for $\mathrm{C}_{23} \mathrm{H}_{22} \mathrm{O}_{4}: \mathrm{C}$, 73.59 ; H. $6.8 \%$.

3-[(3-Benzyloxy-2,2-dimethyl-7-methoxychroman-6-yl)hydroxymethyl]-4-vinyldihydro-2(3H)-furanones (7). To a stirred and cooled $(-70$ " $\mathrm{C})$ solution of potasssium bis(trimethylsilyl)amide (35.6 ml, a $0.5 \mathrm{M}$ sol. in toluene, $17.8 \mathrm{mmol})$ in dry THF $(25 \mathrm{ml})$ under $\mathrm{N}_{2}$ atmosphere. After stirring the mixture at $-75^{\circ} \mathrm{C}$ for $30 \mathrm{~mm}$., a solution of $6(3.24$ g, $9.94 \mathrm{mmol})$ in dry THF $(100 \mathrm{ml})$ was added as drops. The mixture was stirred at $-75^{\circ} \mathrm{C}$ for $8 \mathrm{hr}$, and sat. metanolic $\mathrm{NH}_{4} \mathrm{Cl}$ solution $(5 \mathrm{ml})$ and sat. $\mathrm{NH}_{4} \mathrm{Cl}$ solution $(150 \mathrm{ml})$ were added sequentially. The mixture was allowed to reach room temperature and extracted by EtOAc. The extract was washed by brine, dried over $\mathrm{Na}_{2} \mathrm{SO}_{4}$, filtered and concentrated. Silica-gel chromatography of the residue gave an yellow oily product, and crystallization of the product from diisopropyl ether afforded erythro-7 $(3.03 \mathrm{~g}, 70 \%)$ as white crystals. mp. $102-104^{\circ} \mathrm{C}$. NMR $\delta \mathrm{H}\left(\mathrm{CDCl}_{3}\right): 1.28(1.5 \mathrm{H}, \mathrm{S}), 1.37(3 \mathrm{H}, \mathrm{S}), 2.60$ $(0.5 \mathrm{H}, \mathrm{d}, J=5.37 \mathrm{~Hz}), 2.67(0.5 \mathrm{H}, \mathrm{d}, J=5.37 \mathrm{~Hz}), 2.70-2.77(1 \mathrm{H}, \mathrm{m}), 2.95(1 \mathrm{H}, \mathrm{dt}, J=5.37$, $16.0 \mathrm{~Hz}), 3.04(1 \mathrm{H}, \mathrm{m}), 3.25(1 \mathrm{H}, \mathrm{dd}, J=8.79,17.58 \mathrm{~Hz}), 3.52(1 \mathrm{H}, \mathrm{dd}, J=4.88,7.81 \mathrm{~Hz})$, $3.74(3 \mathrm{H}, \mathrm{S}), 3.87(1 \mathrm{H}, \mathrm{dd}, J=8.79,17.58 \mathrm{~Hz}), 4.34(1 \mathrm{H}, \mathrm{dt}, J=1.96,8.79 \mathrm{~Hz}), 4.54(1 \mathrm{H}, \mathrm{d}$, $J=12.21 \mathrm{~Hz}), 4.67-4.79(3 \mathrm{H}, \mathrm{m}), 5.25-5.38(1 \mathrm{H}, \mathrm{m}), 5.52(1 \mathrm{H}, \mathrm{m}), 6.31(1 \mathrm{H}, \mathrm{S}), 7.12(1 \mathrm{H}$, S), 7.28-7.35 (5H, m). Anal. Found: C, 71.12; H, 6.89. Calcd. for $\mathrm{C}_{25} \mathrm{H}_{30} \mathrm{O}_{6}:$ C, 71.2; H. 6.9\%.

3-[(3-Benzyloxy-2,2-dimethyl-7-methoxychroman-6-yl)tert-butyldimethylsilyloxymethyl]-4-vinyldihydro-2(3H)-furanones $(8)$. To a cooled $\left(0^{\circ} \mathrm{C}\right)$ and stirred solution of 7 
(2.0 g, $4.57 \mathrm{mmol})$ and 2,6-lutidine $(1.6 \mathrm{ml}, 13.7 \mathrm{mmol})$ in dry $\mathrm{CH}_{2} \mathrm{Cl}_{2}(30 \mathrm{ml})$ was added tert-butyldimethylsilyl trifluoromethanesulfonate (TBDMSOTf; $1.57 \mathrm{ml}, 6.84 \mathrm{mmol}$ ) as drops. The solution was stirred at room temperature for $1 \mathrm{hr}$ before adding $5 \% \mathrm{NaHCO}_{3}$ solution, and extracted by $\mathrm{CH}_{2} \mathrm{Cl}_{2}$. The extract was washed with brine, dried over $\mathrm{Na}_{2} \mathrm{SO}_{4}$, filtered and concentrated. The residue was purified by silica-gel chromatography (eluent; hexane : EtOAc $=3: 1)$ to obtain $8(2.14 \mathrm{~g}, 85 \%)$ as colourless crystals. mp. 105-108 "C. $\mathrm{NMR} \delta \mathrm{H}\left(\mathrm{CDCl}_{3}\right) \quad:-0.08(1.2 \mathrm{H}, \mathrm{S}),-0.07(1.8 \mathrm{H}, \mathrm{S}), 0.09(1.2 \mathrm{H}, \mathrm{S}), 0.10(1.8 \mathrm{H}, \mathrm{S}), 0.93$ (3.6H, S), $0.94(5.4 \mathrm{H}, \mathrm{S}), 1.25(1.8 \mathrm{H}, \mathrm{S}), 1.29(1.2 \mathrm{H}, \mathrm{S}), 1.37$ (1.2H, S), 1.39 (1.8H, S), 2.67-2.74 $(1 \mathrm{H}, \mathrm{m}), 2.82-2.86(1 \mathrm{H}, \mathrm{m}), 2.89-2.96(1 \mathrm{H}, \mathrm{m}), 3.29-3.37(1 \mathrm{H}, \mathrm{m}), 3.52-3.57(1 \mathrm{H}$, $\mathrm{m}), 3.74(3 \mathrm{H}, \mathrm{S}), 3.85-3.93(1 \mathrm{H}, \mathrm{m}), 4.35-4.41(1 \mathrm{H}, \mathrm{m}), 4.53-4.47(4 \mathrm{H}, \mathrm{m}), 5.22-5.35(1 \mathrm{H}$, m), $5.56(1 \mathrm{H}, \mathrm{m}), 6.28(0.6 \mathrm{H}, \mathrm{S}), 6.29(0.4 \mathrm{H}, \mathrm{S}), 7.07(0.6 \mathrm{H}, \mathrm{S}), 7.08(0.4 \mathrm{H}, \mathrm{S}), 7.29-7.36$ $(5 \mathrm{H}, \mathrm{m})$. Anal . Found: C, 69.6; H, 8.09. Calcd. for $\mathrm{C}_{32} \mathrm{H}_{44} \mathrm{O}_{6} \mathrm{Si}: \mathrm{C}, 69.53 ; \mathrm{H}, 8.03 \%$.

2-[(3-Benzyloxy-2,2-dimethy]-7-methoxychroman-6-yl)tert-butyldimethylsilyloxymethyl]-3-vinyl-1,4-butanediols (9). To a cooled (-10 "C) and stirred suspension of $\mathrm{LiAlH}_{4}$ $(0.17 \mathrm{~g}, 5.0 \mathrm{mmol})$ in dry THF $(30 \mathrm{ml})$ was added a solution of $8(2.12 \mathrm{~g}, 3.84 \mathrm{mmol})$ in dry THF $(30 \mathrm{ml})$. The mixture was stirred at $-10^{\circ} \mathrm{C}$ for $2 \mathrm{hr}$ before quenching by sat. $\mathrm{MgSO}_{4}$ solution and anhydrous $\mathrm{K}_{2} \mathrm{CO}_{3}$ and stirred for an additional period of $\mathrm{h}$, and filtered through a celite pad. The filtrate was concentrated. The residue was purified by silica-gel chromatography (eluent; hexane : EtOAc $=2: 1)$ to obtain $9(1.93 \mathrm{~g}, 90 \%)$ as a colourless syrup. NMR $\delta \mathrm{H}\left(\mathrm{CDCl}_{3}\right):-0.31(1.2 \mathrm{H}, \mathrm{S}),-0.30(1.8 \mathrm{H}, \mathrm{S}),-0.01(3 \mathrm{H}, \mathrm{S}), 0.84$ (9H, S), 1.27 (1.8H, S), $1.28(1.2 \mathrm{H}, \mathrm{S}), 1.36(3 \mathrm{H}, \mathrm{S}), 2.68-2.76(1 \mathrm{H}, \mathrm{m}), 2.80$ (1H, br. S), $2.86(1 \mathrm{H}, \mathrm{d}, J=4.88 \mathrm{~Hz}), 2.93(1 \mathrm{H}, \mathrm{dt}, J=4.88,16.11 \mathrm{~Hz}), 3.38(1 \mathrm{H}, \mathrm{d}, J=9.79 \mathrm{~Hz}), 3.51-$ $3.55(1 \mathrm{H}, \mathrm{m}), 3.78(1.2 \mathrm{H}, \mathrm{S}), 3.79(1.8 \mathrm{H}, \mathrm{S}), 3.82-3.85(1 \mathrm{H}, \mathrm{m}), 3.96(1 \mathrm{H}$, br. S), $4.50-4.56$ $(1 \mathrm{H}, \mathrm{m}), 4.67-4.73(1 \mathrm{H}, \mathrm{m}), 5.09-5.14(2 \mathrm{H}, \mathrm{m}), 5.22(1 \mathrm{H}, \mathrm{d}, J=8.30 \mathrm{~Hz}), 5.94-6.03(1 \mathrm{H}$, m), $6.34(0.6 \mathrm{H}, \mathrm{S}), 6.35(0.4 \mathrm{H}, \mathrm{S}), 7.06(0.6 \mathrm{H}, \mathrm{S}), 7.10(0.4 \mathrm{H}, \mathrm{S}), 7.27-7.33(5 \mathrm{H}, \mathrm{m})$. Anal . Found: $\mathrm{C}, 69.21 ; \mathrm{H}, 8.56$. Calcd. for $\mathrm{C}_{31} \mathrm{H}_{46} \mathrm{O}_{7} \mathrm{Si}: \mathrm{C}, 69.02 ; \mathrm{H}$. 8.3\%.

4-[3-Benzyloxy-2,2-dimethyl-7-methoxychroman-6-y])tert-butyldimethylsilyloxymethyl]-2-hydroxy-3-hydroxymethyltetrahydrofurans (10) . A mixture of 9 (0.47 g, 0.85 mmol), $N$-methylmorpholine $N$-oxid (NMO) $(0.12 \mathrm{~g}, 1.02 \mathrm{mmol}), 2 \% \mathrm{OsO}_{4}$ solution $(0.5$ $\mathrm{ml})$, acetone $(6 \mathrm{ml})$ and $\mathrm{H}_{2} \mathrm{O}(3 \mathrm{ml})$ in tert-BuOH $(3 \mathrm{ml})$ was stirred at room temperature for $12 \mathrm{hr}$ under $\mathrm{N}_{2}$ atmosphere in the dark. To the reaction mixture was added NaHSO: solution $\left(0.02 \mathrm{~g}\right.$ in $\left.1 \mathrm{ml} \mathrm{H} \mathrm{H}_{2} \mathrm{O}\right)$. After filtering the mixture through a celite pad, the filtrate was concentrated. The residue was dissolved in EtOAc $(10 \mathrm{ml})$, and $\mathrm{NaIO}_{4}(0.20 \mathrm{~g}, 0.94$ mmol) in $\mathrm{H}_{2} \mathrm{O}(5 \mathrm{ml})$ was added to the solution, and the mixture was stirred at room temperature for $24 \mathrm{hr}$. The reaction mixture was extracted by EtOAc. The extract was washed with brine, dried over $\mathrm{Na}_{2} \mathrm{SO}_{4}$, filtered and concentrated. The residue was purified by silica-gel chromatography (eluent; hexane :EtOAc=1:2) to obtain $10 \quad(0.403$ g, $85 \%$ ) as colourless crystals. mp. $61-64^{\circ} \mathrm{C}$. Anal. Found: C, 66.63; H, 8.37. Calcd. for $\mathrm{C}_{31} \mathrm{H}_{46} \mathrm{O}_{7} \mathrm{Si}: \mathrm{C}, 66.63 ; \mathrm{H}, 8.3 \%$.

4-[3-Benzyloxy-2,2-dimethyl-7-methoxychroman-6-yl)tert-butyldimethylsilyloxymethyl]-3-hydroxymethyldihydro-2(3H)-furanones (11) . The mixture of $10 \quad(1.25 \mathrm{~g}, 2.24$ mmol) and $\mathrm{Ag}_{2} \mathrm{CO}_{3}$-celite (2.24 g containing $2.24 \mathrm{mmol}$ of $\left.\mathrm{Ag}_{2} \mathrm{CO}_{3}\right)$ in benzene $(20 \mathrm{ml})$ was refluxed for $30 \mathrm{~min}$. The reaction mixture was filtered, and the filtrate was concentrated. The residue was purified by silica-gel chromatography (eluent; hexane :EtOAc $=3: 1$ ) to obtain 11 (1.21 g, 97\%) as colourless crystals. mp. 48-52 "C. NMR $\delta \mathrm{H}\left(\mathrm{CDCl}_{3}\right):-0.31$ 
(1.8H, S), - 0.29 (1.2H, S), $0.54(3 \mathrm{H}, \mathrm{S}), 0.87$ (9H, S), 1.24 (1.2H, S), 1.26 (1.8H, S), 1.28 $(1.2 \mathrm{H}, \mathrm{S}), 1.29(1.8 \mathrm{H}, \mathrm{S}), 2.67-2.99(5 \mathrm{H}, \mathrm{m}), 3.10-3.15(1 \mathrm{H}, \mathrm{m}), 3.51-3.56(1 \mathrm{H}, \mathrm{m}), 3.75$ $(3 \mathrm{H}, \mathrm{S}), 3.93-4.16(5 \mathrm{H}, \mathrm{m}), 4.55(1 \mathrm{H}, \mathrm{dd}, J=9.28,11.78 \mathrm{~Hz}), 4.71(1 \mathrm{H}, \mathrm{dd}, J=5.86,11.78$ $\mathrm{Hz}), 5.18(1 \mathrm{H}, \mathrm{m}), 6.32(0.6 \mathrm{H}, \mathrm{S}), 6.33(0.4 \mathrm{H}, \mathrm{S}), 7.02(0.6 \mathrm{H}, \mathrm{S}), 7.04(0.4 \mathrm{H}, \mathrm{S}), 7.33-7.35$ (5H, m). Anal. Found: C, 66.65; H, 7.97. Calcd. for $\mathrm{C}_{31} \mathrm{H}_{46} \mathrm{O}_{7} \mathrm{Si}: \mathrm{C}, 66.87 ; \mathrm{H}, 7.97 \%$.

4-[3-Benzyloxy-2,2-dimethyl-7-methoxychroman-6-yl)tert-butyldimethylsilyloxymethyl]-3-methylenedihydro-2(3H)-furanones (12). To an ice-cooled solution of 11 (1.20 $\mathrm{g}, 2.16 \mathrm{mmol})$ and $\mathrm{Et}_{3} \mathrm{~N}(0.60 \mathrm{ml}, 4.32 \mathrm{mmol})$ in benzene $(15 \mathrm{ml})$ was added methanesulfonyl chloride $(0.25 \mathrm{ml}, 3.24 \mathrm{mmol})$ as drops, and the mixture was stirred at room temperature for $2 \mathrm{hr}$. To the solution was added 1,8-diazabicyclo[5.4.0]undec-7-ene (DBU) $(0.48 \mathrm{ml}, 3.24 \mathrm{mmol})$, and the mixture was stirred at room temperature for $1 \mathrm{hr}$. The reaction mixture was diluted with EtOAc, washed with 5\% citric acid solution, 5\% $\mathrm{NaHCO}_{3}$, brine, dried over $\mathrm{Na}_{2} \mathrm{SO}_{4}$, filtered and concentrated. The residue was purified by silica-gel chromatography (eluent; hexane :EtOAc=4:1) to obtain $12(0.91 \mathrm{~g}, 78 \%)$ as a

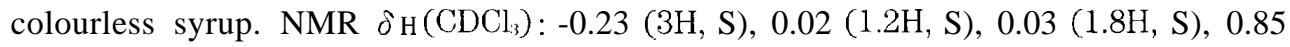
(3.6H, S), $0.86(5.4 \mathrm{H}, \mathrm{S}), 1.29$ (1.2H, S), $1.30(1.8 \mathrm{H}, \mathrm{S}), 1.37(1.8 \mathrm{H}, \mathrm{S}), 1.38(1.2 \mathrm{H}, \mathrm{S})$, 2.66-2.74 (1H, m), 2.86-3.00 (1H, m), $3.23(1 \mathrm{H}, \mathrm{m}), 3.54(1 \mathrm{H}, \mathrm{m}), 3.75(3 \mathrm{H}, \mathrm{S}), 4.17-4.29$ $(2 \mathrm{H}, \mathrm{m}), 4.54(1 \mathrm{H}, \mathrm{m}), 4.71(1 \mathrm{H}, \mathrm{m}), 5.07(1 \mathrm{H}, \mathrm{m}), 5.14(0.6 \mathrm{H}, \mathrm{S}), 5.23(0.4 \mathrm{H}, \mathrm{S}), 6.22$ $(1 \mathrm{H}, \mathrm{d}, J=5.38 \mathrm{~Hz}), 6.33(1 \mathrm{H}, \mathrm{S}), 6.96(0.6 \mathrm{H}, \mathrm{S}), 6.99(0.4 \mathrm{H}, \mathrm{S}), 7.27-7.35(5 \mathrm{H}, \mathrm{m})$. Anal. Found: C, 68.84; $\mathrm{H}, 7.89$. Calcd. for $\mathrm{C}_{31} \mathrm{H}_{43} \mathrm{O}_{6} \mathrm{Si}: \mathrm{C}, 69.11 ; \mathrm{H}, 7.86 \%$.

4-[3-Benzyloxy-2,2-dimethyl-7-methoxychroman-6-yl)tert-butyldimethylsilyloxymethyl]-3-hydroxy-3-hydroxymethyldihydro-2(3H)-furanones (13). A mixture of 12 (0.90 $\mathrm{g}, 1.67 \mathrm{mmol}), 2 \% \mathrm{OsO}_{4}$ solution $(0.5 \mathrm{ml})$, NMO $(0.22 \mathrm{~g}, 1.88 \mathrm{mmol})$, acetone $(12 \mathrm{ml})$, tert-BuOH $(6 \mathrm{ml})$ and $\mathrm{H}_{2} \mathrm{O}(6 \mathrm{ml})$ was stirred at room temperature for $12 \mathrm{hr}$ under $\mathrm{N}_{2}$ atmosphere. To the mixture was added $\mathrm{NaHSO}_{3}$ solution $\left(0.13 \mathrm{~g}\right.$, in $\left.1 \mathrm{ml} \mathrm{H}_{2} \mathrm{O}\right)$. After filtering through a celite pad, the filtrate was concentrated. The residue was purified by silica-gel chromatography (eluent; hexane :EtOAc $=2: 1)$ to obtain $13(0.80 \mathrm{~g}, 83 \%)$ as a colourless syrup. NMR $\delta \mathrm{H}\left(\mathrm{CDCl}_{3}\right): 1.29(3 \mathrm{H}, \mathrm{S}), 1.37(3 \mathrm{H}, \mathrm{S}), 2.71(1 \mathrm{H}, \mathrm{dd}, J=7.32$, $16.11 \mathrm{~Hz}), 2.92(2 \mathrm{H}, \mathrm{dd}, J=4.88,16.11 \mathrm{~Hz}), 3.06(1 \mathrm{H}, \mathrm{dd}, J=5.86,13.18 \mathrm{~Hz}), 3.12-3.21$ $(1 \mathrm{H}, \mathrm{m}), 3.36(1 \mathrm{H}, \mathrm{S}), 3.50-3.54(1 \mathrm{H}, \mathrm{m}), 3.79(3 \mathrm{H}, \mathrm{S}), 3.81-4.11(5 \mathrm{H}, \mathrm{m}), 4.55(1 \mathrm{H}, \mathrm{d}$, $J=12.21 \mathrm{~Hz}), 4.71(1 \mathrm{H}, \mathrm{d}, J=12.21 \mathrm{~Hz}), 5.04-5.09(1 \mathrm{H}, \mathrm{m}), 6.37(1 \mathrm{H}, \mathrm{S}), 6.90(6 \mathrm{H}, \mathrm{S}), 6.98$ $(0.4 \mathrm{H}, \mathrm{S}), 7.28-7.37(5 \mathrm{H}, \mathrm{m})$. Anal. Found: C, 65.45; H, 7.68. Calcd. for $\mathrm{C}_{31} \mathrm{H}_{44} \mathrm{O}_{8} \mathrm{Si}: \mathrm{C}$, $65.0 ; \mathrm{H}, 7.75 \%$.

4-[3-Benzyloxy-2,2-dimethyl-7-methoxychroman-6-yl)hydroxymethyl]-3-hydroxy-3hydroxymethyldihydro-2 $(3 H)$-furanones $(14)$. To a cooled $\left(0^{\circ} \mathrm{C}\right)$ solution of $13(0.79 \mathrm{~g}$, $1.38 \mathrm{mmol})$ in dry THF $(10 \mathrm{ml})$ was slowly added tetrabutylammonium fluoride (TBAF; $1.66 \mathrm{ml}$, a $1.0 \mathrm{M}$ solution in THF, $1.66 \mathrm{mmol}$ ), and stirred at $0^{\circ} \mathrm{C}$ for $1.5 \mathrm{hr}$. The reaction mixture was diluted with EtOAc, washed with $10 \% \mathrm{NH}_{4} \mathrm{Cl}$ solution, brine, dried over $\mathrm{Na}_{2} \mathrm{SO}_{4}$, filtered and concentrated. The residue was purified by silica-gel chromatography (eluent; hexane :EtOAc=1:2) to obtain $14(0.44 \mathrm{~g}, 70 \%)$ as colourless crystals. mp. 146$149^{\circ} \mathrm{C}$. NMR $\delta \mathrm{H}\left(\mathrm{CDCl}_{3}\right): 1.29(3 \mathrm{H}, \mathrm{S}), 1.37(3 \mathrm{H}, \mathrm{S}), 2.72(1 \mathrm{H}, \mathrm{dd}, J=7.32,16.11 \mathrm{~Hz}), 2.93$ $(2 \mathrm{H}, \mathrm{dd}, J=4.88,16.11 \mathrm{~Hz}), 3.05(1 \mathrm{H}, \mathrm{dd}, J=5.86,13.18 \mathrm{~Hz}), 3.12-3.21(1 \mathrm{H}, \mathrm{m}), 3.36(1 \mathrm{H}$, S), 3.50-3.54 (1H, m), 3.79 (3H, S), $3.88(1 \mathrm{H}, \mathrm{dt}, J=2.93,9.28 \mathrm{~Hz}), 3.96(1 \mathrm{H}, \mathrm{m}), 4.03(1 \mathrm{H}$, dd, $J=5.86,11.72 \mathrm{~Hz}), 4.09(1 \mathrm{H}, \mathrm{dd}, J=5.37,11.72 \mathrm{~Hz}), 4.55(1 \mathrm{H}, \mathrm{d}, J=12.21 \mathrm{~Hz}), 4.71$ $(1 \mathrm{H}, \mathrm{d}, J=12.21 \mathrm{~Hz}), 5.06(1 \mathrm{H}, \mathrm{m}), 6.37(1 \mathrm{H}, \mathrm{S}), 6.97(1 \mathrm{H}, \mathrm{S}), 7.28-7.35(5 \mathrm{H}, \mathrm{m})$. Anal. 
Found: $\mathrm{C}, 65.21 ; \mathrm{H}, 6.65$. Calcd. for $\mathrm{C}_{25} \mathrm{H}_{30} \mathrm{O}_{8}: \mathrm{C}, 65.47 ; \mathrm{H}, 6.60 \%$.

$6 \alpha$-(3-Benzyloxy-2,2-dimethyl-7-methoxychroman-6-yl)-1 $\alpha$-hydroxy-2-oxo-3,7dioxabicyclo[3.3.0]octanes (15). To an ice-cooled solution of $14(0.43 \mathrm{~g}, 0.94 \mathrm{mmol})$ in dry $\mathrm{CH}_{2} \mathrm{Cl}_{2}(20 \mathrm{ml})$ was added a catalytic amount of 10-camphorsurfonic acid, and the mixture was stirred at $0{ }^{\circ} \mathrm{C}$ for $1.5 \mathrm{hr}$ before quenching with a few drops of $\mathrm{Et}_{3} \mathrm{~N}$. The solvent was evaporated. The residue was purified by silica-gel chromatography (eluent; hexane: EtOAc $=2: 1)$ to obtain $15(0.353 \mathrm{~g}, 86 \%)$ as colourless crystals. mp. $62-65{ }^{\circ} \mathrm{C}$.

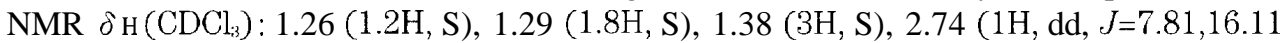
$\mathrm{Hz}), 2.96(1 \mathrm{H}, \mathrm{S}), 2.93-2.99(1 \mathrm{H}, \mathrm{m}), 3.12(1 \mathrm{H}, \mathrm{m}), 3.53(1 \mathrm{H}, \mathrm{m}), 3.76(3 \mathrm{H}, \mathrm{S}), 4.12-4.22$ $(2 \mathrm{H}, \mathrm{m}), 4.37-4.42(1 \mathrm{H}, \mathrm{m}), 4.47-4.67(2 \mathrm{H}, \mathrm{m}), 4.72(1 \mathrm{H}, \mathrm{d}, J=11.72 \mathrm{~Hz}), 5.08(1 \mathrm{H}, \mathrm{d}$, $J=4.89 \mathrm{~Hz}), 6.36(1 \mathrm{H}, \mathrm{S}), 7.15(1 \mathrm{H}, \mathrm{S}), 7.27-7.35(5 \mathrm{H}, \mathrm{m})$. Anal. Found: C, 67.68; H, 6.50. Calcd. for $\mathrm{C}_{25} \mathrm{H}_{28} \mathrm{O}_{7}: \mathrm{C}, 68.15$; H. $6.41 \%$.

$6 \alpha$-(3-Benzyloxy-2,2-dimethyl-7-methoxychroman-6-yl)-1 $\alpha$-tert-butyldimethylsilyloxy-2-oxo-3,7-dioxabicyclo[3.3.0]octanes (16). To a cooled (0 "C) solution of $15(0.34$ $\mathrm{g}, 0.77 \mathrm{mmol})$ and 2,6 -lutidine $(0.27 \mathrm{ml}, 2.32 \mathrm{mmol})$ in dry $\mathrm{CH}_{2} \mathrm{Cl}_{2}(10 \mathrm{ml})$ was added tertbutyldimethylsilyl trifluoromethanesulfonate (TBDMSOTf) $(0.27 \mathrm{ml}, 1.18 \mathrm{mmol})$ by a syringe. The solution was stirred at room temperature for $24 \mathrm{hr}$. After $24 \mathrm{hr}$, the same amount of 2,6-lutidine and TBDMSOTf were added to the mixture, and stirred at room temperature for $24 \mathrm{hr}$ before quenching by an addition of $5 \% \mathrm{NaHCO}_{3}$ solution. The organic layer was separated, and the aqueous layer was extracted by $\mathrm{CH}_{2} \mathrm{Cl}_{2}$. The combined organic layers were washed with brine, dried over $\mathrm{Na}_{2} \mathrm{SO}_{4}$, filtered and concentrated. The residue was purified by silica-gel chromatography (eluent; hexane : EtOAc $=4: 1)$ to obtain $16(0.37 \mathrm{~g}, 86 \%)$ as a colourless syrup. NMR $\delta \mathrm{H}\left(\mathrm{CDCl}_{3}\right): 0.02$ $(1.8 \mathrm{H}, \mathrm{S}), 0.03(1.2 \mathrm{H}, \mathrm{S}), 0.17(1.8 \mathrm{H}, \mathrm{S}), 0.18(1.2 \mathrm{H}, \mathrm{S}), 0.78(3.6 \mathrm{H}, \mathrm{S}), 0.79(5.4 \mathrm{H}, \mathrm{S})$, $1.28(1.2 \mathrm{H}, \mathrm{S}), 1.29(1.2 \mathrm{H}, \mathrm{S}), 1.38(1.2 \mathrm{H}, \mathrm{S}), 1.39(1.8 \mathrm{H}, \mathrm{S}), 2.73(1 \mathrm{H}, \mathrm{dt}, J=7.81,16.6$ $\mathrm{Hz}), 2.93(1 \mathrm{H}, \mathrm{dd}, J=5.37,16.11 \mathrm{~Hz}), 3.01(1 \mathrm{H}, \mathrm{m}), 3.52(1 \mathrm{H}, \mathrm{m}), 3.75(3 \mathrm{H}, \mathrm{S}), 4.10-4.16$ $(1 \mathrm{H}, \mathrm{m}), 4.23(1 \mathrm{H}, \mathrm{dd}, J=3.41,9.76 \mathrm{~Hz}), 4.34(1 \mathrm{H}, \mathrm{dd}, J=1.95,4.88 \mathrm{~Hz}), 4.53-4.61(2 \mathrm{H}$, m), $4.72(1 \mathrm{H}, \mathrm{d}, J=11.72 \mathrm{~Hz}), 5.07(1 \mathrm{H}, \mathrm{d}, J=4.40 \mathrm{~Hz}), 6.35(0.6 \mathrm{H}, \mathrm{S}), 6.36(0.4 \mathrm{H}, \mathrm{S}), 7.11$ $(1 \mathrm{H}, \mathrm{S})$. Anal. Found: C, 66.85; H, 7.70. Calcd. for $\mathrm{C}_{31} \mathrm{H}_{43} \mathrm{O}_{7} \mathrm{Si}: \mathrm{C}, 67.12 ; \mathrm{H}$. 7.64\%.

$1 \alpha$-tert-Butyldimethylsilyloxy-6 $\alpha$-(2,2-dimethyl-3-hydroxy-7-methoxychroman-6-yl)2-oxo-3,7-dioxabicyclo[3.3.0]octanes (17). A mixture of 16 (0.344 g, $0.62 \mathrm{mmol})$ and 20\% $\mathrm{Pd}(\mathrm{OH})_{2}-\mathrm{C}(0.25 \mathrm{~g})$ in EtOAc $(50 \mathrm{ml})$ was vigorously stirred for $10 \mathrm{~min}$ under $\mathrm{H}_{2}$ atmosphere. The suspension was filtered, and the filtrate was concentrated. The residue was purified by silica-gel chromatography (eluent; hexane :EtOAc=2:1) to obtain 17 $(0.254 \mathrm{~g}, 88 \%)$ as colourless crystals. mp. 103-106 ${ }^{\circ} \mathrm{C} . \mathrm{NMR} \delta \mathrm{H}\left(\mathrm{CDCl}_{3}\right): 0.02(1.2 \mathrm{H}, \mathrm{S})$, 0.03 (1.8H, S), 0.17 (3H, S), 0.77 (3.6H, S), 0.79 (5.4H, S), 1.31 (3H, S), 1.36 (3H, S), 1.67 $(1 \mathrm{H}, \mathrm{d}, J=7.81 \mathrm{~Hz}), 2.67-2.75(1 \mathrm{H}, \mathrm{m}), 2.97-3.04(2 \mathrm{H}, \mathrm{m}), 3.77(3 \mathrm{H}, \mathrm{S}), 3.78-3.80(1 \mathrm{H}, \mathrm{m})$, $4.14(0.6 \mathrm{H}, \mathrm{d}, J=9.77 \mathrm{~Hz}), 4.24(0.4 \mathrm{H}, \mathrm{d}, J=9.77 \mathrm{~Hz}), 4.34(1 \mathrm{H}, \mathrm{m}), 4.59(1 \mathrm{H}, \mathrm{m}), 5.08$ $(1 \mathrm{H}, \mathrm{m}), 6.38(1 \mathrm{H}, \mathrm{S}), 7.13(0.4 \mathrm{H}, \mathrm{S}), 7.14(0.6 \mathrm{H}, \mathrm{S})$. Anal. Found: C, 62.0; H, 7.84. Calcd. for $\mathrm{C}_{24} \mathrm{H}_{36} \mathrm{O}_{7} \mathrm{Si}: \mathrm{C}, 62.04$; H. 7.82\%.

$1 \alpha$-tert-Butyldimethylsilyloxy-6 $\alpha$-(2,2-dimethyl-7-methoxychromen-6-yl)-2-oxo-3,7dioxabicyclo[3.3.0]octane (18). To an ice-cooled solution of $17(53.4 \mathrm{mg}, 0.115 \mathrm{mmol})$ and 4-dimethylaminopyridine $(0.10 \mathrm{~g}, 0.82 \mathrm{mmol})$ in dry $\mathrm{CH}_{2} \mathrm{Cl}_{2}(4 \mathrm{ml})$ was added methanesulfonyl chloride $(53.4 \mu \mathrm{l}, 0.69 \mathrm{mmol})$ by a micro syringe, and stirred at room temperature for $48 \mathrm{hr}$. To the reaction mixture was added $\mathrm{H}_{2} \mathrm{O}$, acidified by $5 \%$ citric acid 
solution and extracted by $\mathrm{CH}_{2} \mathrm{Cl}_{2}$. The extract was washed with sat. $\mathrm{NaHCO}$, brine, dried over $\mathrm{Na}_{2} \mathrm{SO}_{4}$, filtered and concentrated. The residue was purified by preparative TLC (1.0 $\mathrm{mm}$ thickness, eluent; hexane :EtOAc $=2: 1)$ to obtain a mesylate $(61.0 \mathrm{mg}, 98 \%)$ as a colourless oil. To a solution of mesylate $(37.5 \mathrm{mg}, 0.069 \mathrm{mmol})$ in toluene $(3 \mathrm{ml})$ was added DBU $(0.10 \mathrm{ml}, 0.67 \mathrm{mmol})$, and refluxed for $12 \mathrm{~h}$. The solution was diluted by benzene, washed with $5 \%$ citric acid solution, sat. $\mathrm{NaHCO}$,, brine, dried over $\mathrm{Na}_{2} \mathrm{SO}_{4}$, filtered and evaporated. The residue was purified by preparative TLC $(0.25 \mathrm{~mm}$ thickness, eluent; hexane: $\mathrm{EtOAc}=2: 1)$ to obtain $18(22.2 \mathrm{mg}, 72 \%)$ as colourless crystals. mp. 106-109 ${ }^{\circ} \mathrm{C}$. NMR $\delta \mathrm{H}\left(\mathrm{CDCl}_{3}\right): 0.04(3 \mathrm{H}, \mathrm{S}), 0.17(3 \mathrm{H}, \mathrm{S}), 0.80(9 \mathrm{H}, \mathrm{S}), 1.42$ (3H, S), 1.43 (3H, S), $3.00(1 \mathrm{H}, \mathrm{m}), 3.79(3 \mathrm{H}, \mathrm{S}), 4.13(1 \mathrm{H}, \mathrm{d}, J=10.26 \mathrm{~Hz}), 4.34(1 \mathrm{H}, \mathrm{dd}$, $J=4.88,9.77 \mathrm{~Hz}), 4.59(1 \mathrm{H}, \mathrm{dd}, J=7.81,9.77 \mathrm{~Hz}), 5.07(1 \mathrm{H}, \mathrm{d}, J=4.39 \mathrm{~Hz}), 5.48(1 \mathrm{H}, \mathrm{d}$, $J=9.77 \mathrm{~Hz}), 6.28(1 \mathrm{H}, \mathrm{d}, J=9.77 \mathrm{~Hz}), 6.37(1 \mathrm{H}, \mathrm{S}), 7.07(1 \mathrm{H}, \mathrm{S})$. Anal. Found: C, 64.67; $\mathrm{H}, 7.61$. Calcd. for $\mathrm{C}_{24} \mathrm{H}_{34} \mathrm{O}_{6} \mathrm{Si}: \mathrm{C}, 64.54 ; \mathrm{H} .7 .68 \%$.

$6 \alpha$-(2,2-Dimethyl-7-methoxychromen-6-yl)-1 a-hydroxy-2-oxo-3,7-dioxabicyclo[3.3.0]octane (19). To a solution of $18(35.5 \mathrm{mg}, 0.079 \mathrm{mmol})$ in dry THF (3 ml) was added TBAF $(0.12 \mathrm{ml}$, a $1.0 \mathrm{M}$ solution in THF, $0.12 \mathrm{mmol})$ by a micro syringe, and stirred at room temperature for $\mathrm{lhr}$. The reaction mixture was quenched by $10 \% \mathrm{NH}_{4} \mathrm{Cl}$ solution, and extracted by EtOAc. The extract was washed with brine, dried over $\mathrm{Na}_{2} \mathrm{SO}_{4}$, filtered and concentrated. The residue was purified by preparative TLC $(0.5 \mathrm{~mm}$ thickness, eluent; hexane : EtOAc=1 : 2) to obtain $20(12.0 \mathrm{mg}, 63 \%)$ as colourless crystals. $\mathrm{mp}$. 149-151 “C. NMR $\delta \mathrm{H}\left(\mathrm{CDCl}_{3}\right): 1.43(6 \mathrm{H}, \mathrm{S}), 2.90(1 \mathrm{H}$, br. S), $3.03(1 \mathrm{H}, \mathrm{m}), 3.79(3 \mathrm{H}, \mathrm{S})$, $4.18(2 \mathrm{H}, \mathrm{S}), 4.39(1 \mathrm{H}, \mathrm{dd}, J=5.37,9.77 \mathrm{~Hz}), 4.65(1 \mathrm{H}, \mathrm{m}), 5.09(1 \mathrm{H}, \mathrm{d}, J=4.39 \mathrm{~Hz}), 5.48$ $(1 \mathrm{H}, \mathrm{d}, J=9.77 \mathrm{~Hz}), 6.29(1 \mathrm{H}, \mathrm{d}, J=9.77 \mathrm{~Hz}), 6.37(1 \mathrm{H}, \mathrm{S}), 7.11(1 \mathrm{H}, \mathrm{S})$, Anal. Found: C, 64.95; $\mathrm{H}, 6.28$. Calcd. for $\mathrm{C}_{18} \mathrm{H}_{20} \mathrm{O}_{6}: \mathrm{C}, 65.04 ;$ H. $6.07 \%$.

$1 \alpha, 2 \alpha / \beta$-Dihydroxy-6 $\alpha$-(2,2-dimethyl-7-methoxychromen-6-yl)-3,7-dioxabicyclo[3.3.0]octanes (20). To a cooled $\left(70^{\circ} \mathrm{C}\right)$ solution of $19(19.1 \mathrm{mg}, 0.058 \mathrm{mmol})$ in dry toluene $(4 \mathrm{ml})$ was added diisobutylaluminium hydride $(0.18 \mathrm{ml}$, a $0.98 \mathrm{M}$ solution in hexane, $0.176 \mathrm{mmol}$ ) under $\mathrm{N}_{2}$ atmosphere, and stirred at same temperature for $2 \mathrm{hr}$. The reaction mixture was quenched by $0.5 \mathrm{~N} \mathrm{HCl}(2 \mathrm{ml})$, and allowed to reach room temperature. The mixture was extracted by EtOAc. The extract was washed with sat. $\mathrm{NaHCO}$, dried over $\mathrm{Na}_{2} \mathrm{SO}_{4}$, filtered and concentrated. The residue was purified by preparative TLC $(0.5 \mathrm{~mm}$ thickness, eluent; hexane : EtOAc=l : 1) to obtain $20(12.0 \mathrm{mg}$, $63 \%)$ as a colourless oil. NMR $\delta \mathrm{H}\left(\mathrm{CDCl}_{3}\right): 1.42(6 \mathrm{H}, \mathrm{S}), 2.52(1 \mathrm{H}, \mathrm{dd}, J=1.95,6.83 \mathrm{~Hz})$, $3.71(1 \mathrm{H}, \mathrm{d}, J=9.76 \mathrm{~Hz}), 3.77(3 \mathrm{H}, \mathrm{S}), 3.80(1 \mathrm{H}$, br. S), $3.97(1 \mathrm{H}, \mathrm{dd}, J=1.95,6.83 \mathrm{~Hz})$, $4.26(1 \mathrm{H}, \mathrm{d}, J=9.77 \mathrm{~Hz}), 4.29(1 \mathrm{H}, \mathrm{dd}, J=6.84,9.28 \mathrm{~Hz}), 4.81(1 \mathrm{H}, \mathrm{d}, J=9.77 \mathrm{~Hz}), 5.19(1 \mathrm{H}$, S), $5.46(1 \mathrm{H}, \mathrm{d}, J=9.77 \mathrm{~Hz}), 6.29(1 \mathrm{H}, \mathrm{d}, J=9.77 \mathrm{~Hz}), 6.35(1 \mathrm{H}, \mathrm{S}), 7.18(1 \mathrm{H}, \mathrm{S})$. Anal. Found: $\mathrm{C}, 64.85 ; \mathrm{H}, 6.59$. Calcd. for $\mathrm{C}_{18} \mathrm{H}_{22} \mathrm{O}_{6}: \mathrm{C}, 64.66 ; \mathrm{H}$. 6.63\%.

$2 \alpha$-(2,6-Dimethoxyphenoxy)-6 $\alpha$-(2,2-dimethyl-7-methoxychromen-6-yl)-1 $\alpha$-hydroxy3,7-dioxabicyclo[3.3.0]octane (21). A solution of $20(12.0 \mathrm{mg}, 0.036 \mathrm{mmol}), 2,6$ dimethoxyphenol $(0.13 \mathrm{~g}, 0.84 \mathrm{mmol})$ and a catalytic amount of pyridinium $p$-toluenesulfonate in benzene $(5 \mathrm{ml})$ was refluxed for $1 \mathrm{hr}$ with azeotropic removal of the water. The reaction mixture was diluted by EtOAc, washed with $1 \mathrm{~N} \mathrm{NaOH}$, brine, dried over $\mathrm{Na}_{2} \mathrm{SO}_{4}$, filtered and concentrated. The residue was purified by preparative TLC $(0.25$ $\mathrm{mm}$ thickness, eluent; hexane :EtOAc $=2: 1)$ to obtain $21(3.7 \mathrm{mg}, 22 \%)$ as a colourless syrup. NMR $\delta \mathrm{H}\left(\mathrm{CDCl}_{3}\right): 1.42\left(3 \mathrm{H}, \mathrm{S}, 2 "-\mathrm{CH}_{3}\right), 1.43\left(3 \mathrm{H}, \mathrm{S}, 2\right.$ "- $\left.\mathrm{CH}_{3}\right), 2.65(1 \mathrm{H}, \mathrm{m}, 5 \alpha-H)$, 
$3.36(1 \mathrm{H}, \mathrm{d}, J=9.76 \mathrm{~Hz}, 8 \alpha-H), 3.77\left(3 \mathrm{H}, \mathrm{S}, 7^{\prime \prime}-\mathrm{OCH}_{3}\right), 3.86\left(6 \mathrm{H}, \mathrm{S}, 2^{\prime}, 66^{\prime}-\mathrm{OCH}_{3}\right), 4.08(1 \mathrm{H}$, dd, $J=1.95,9.28 \mathrm{~Hz}, 4 \alpha-H), 4.25(1 \mathrm{H}, \mathrm{S},-\mathrm{OH}), 4.32(1 \mathrm{H}, \mathrm{d}, J=9.76 \mathrm{~Hz}, 8 \beta-H), 4.59(1 \mathrm{H}$, dd, $J=7.32,9.28 \mathrm{~Hz}, 4 \beta-H), 5.36(1 \mathrm{H}, \mathrm{S}, 2 \beta-H), 5.46(1 \mathrm{H}, \mathrm{d}, J=9.76 \mathrm{~Hz}, 3 "-H), 6.31(1 \mathrm{H}$, d, $J=9.76 \mathrm{~Hz}, 4 "-H), 6.35(1 \mathrm{H}, \mathrm{S}, 6 "-H), 6.60(2 \mathrm{H}, \mathrm{d}, J=8.30 \mathrm{~Hz}, 3,5 '-H), 7.03(1 \mathrm{H}, \mathrm{t}$, $\left.J=8.30 \mathrm{~Hz}, 4^{\prime}-H\right), 7.26(1 \mathrm{H}, \mathrm{S}, 5 "-H)$, Anal. Found: C, 66.51; H, 6.39. Calcd. for $\mathrm{C}_{26} \mathrm{H}_{30} \mathrm{O}_{8}$ : C, 66.37; H. $6.43 \%$.

Bioassay: The precocious metamorphosis activity of 21 was assessed on 10 newly molted 4th instar larva of silkworm (Bombyx mori). One $\mu \mathrm{l}$ of acetone solutions dissolving 40, 80, 160 and $200 \mathrm{\mu g}$ each of the compound were topically applied to the head-thorax by a micro applicator, respectively. The control was tested with $1 \mu \mathrm{l}$ acetone. The activity was evaluated by induction of precocious metamorphosis, spinning a cocoon and subsequent pupation or formation of larval pupal intermediate.

\section{RESULTS AND DISCUSSION}

Synthesis

In the synthesis of a haedoxan analogue possessing precocene I (2,2-dimethyl-7methoxychromene) framework in the molecular structure, protection of the 3,4-olefinic bond of the chromene annoyed us, since the synthetic method of haedoxans involves oxidation reactions using $\mathrm{OsO}_{4}$ at two steps in their syntheses. (Ishibashi and Taniguchi, 1989). After several preliminary experiments, 3-/4-benzyloxychroman-6-carbaldehydes were selected as the starting material to carry out dehydration reaction at a final step of the synthesis. It was readily found that 4-benzyloxy-2,2-dimethyl-7-methoxychroman was easily dehydrated to give 2,2-dimethyl-7-methoxychromen-6-carbaldehyde in Vilsmeierformylation reaction. However, successful formylation was achieved by using 3-benzyloxy -2,2-dimethyl-7-methoxychroman (5), and 3-benzyloxy-2,2-dimethyl-7-methoxychroman-6carbaldehyde (6) was obtained in an $88 \%$ yield.

A haedoxan analogue 2-(2,6-dimethoxyphenoxy)-6-(2,2-dimethyl-7-methoxychromen -6-yl)-1-hydroxy-3,7-dioxabicyclo[3.3.0]octane (21) was synthesized in the following route.

Precocene I (1), which was prepared according to the method in literature (Ohta and Bowers, 1977), was oxidized by $\mathrm{OsO}_{4}-N$-methylmorpholine $N$-oxid (NMO) to obtain a glycol. 2,2-Dimethyl-7-methoxychroman-3,4-diol (2) was converted to 2,2-dimethyl-7methoxychroman-3-one (3) by treatment with conc. HCl solution in tetrahydrofuran (THF). Reduction of 3 with $\mathrm{LiAlH}_{4}$ and benzylation of the product gave 3-benzyloxy-2,2dimethyl-7-methoxychroman (5). Vilsmeier reaction of 5 furnished 3-benzyloxy-2,2dimethyl-7-methoxychroman-6-carbaldehyde (6) in an $88 \%$ yield. In this manner the chromancarbaldehyde was obtained from precocene I by five steps in a $36 \%$ overall yield (Scheme 1).

Similarly to 7 -methoxy-2,2-dimethylchroman-6-carbaldehyde (Gotanda et al., 1993), compound 6 scarcely reacted with the lithium enolate of $\beta$-vinyl- $\gamma$-butyrolactone prepared by using lithium diisopropylamide (LDA) in THF. However, aldol condensation between 6 and potassium enolate of the butyrolactone prepared by using potassium bis(trimethylsilyl)amide (KHMDS) predominantly gave an erythro-aldol (7) in a 70\% yield. 

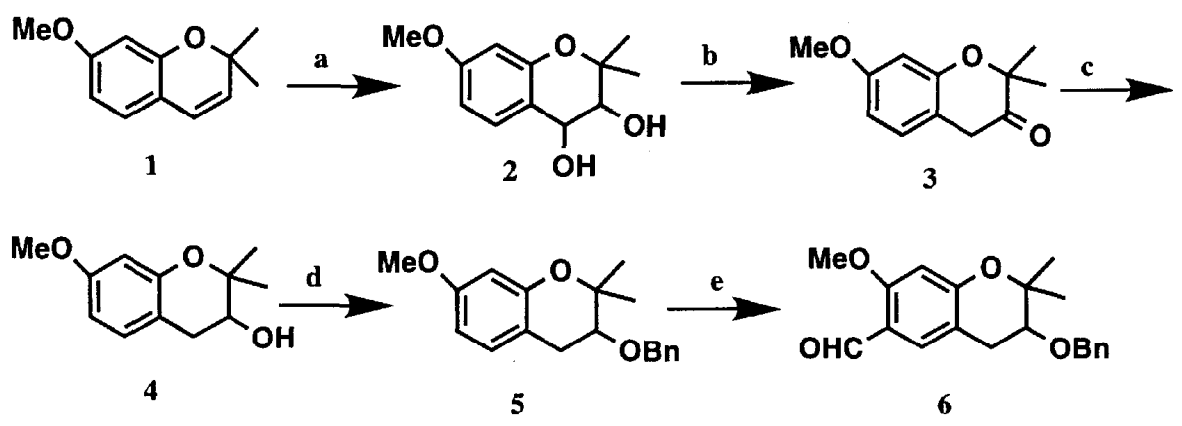

a) cat. $\mathrm{OsO}_{4}$, $\mathrm{NMO} / a q$. acetone, tert-BuOH; b) conc. $\mathrm{HCl} / \mathrm{THF}$

c) $\mathrm{LiAlH}_{4} / \mathrm{THF}$; d) $\mathrm{NaH}, \mathrm{BnBr} / \mathrm{THF}$; e) $\mathrm{POCl}_{3} / \mathrm{DMF}$;

Scheme 1. Synthesis of chroman-6-carbaldehyde.

According to the procedures developed in haedoxan synthesis (Ishibashi and Taniguchi, 1989), compound 7 was converted to a 6-(3-benzyloxy-2,2-dimethyl-7-methoxychroman6-yl)-1-hydroxy-2-oxo-3,7-dioxabicyclo[3.3.0]octane (15) through 8 steps in a $25 \%$ overall yield. Debenzylation by catalytic hydrogenation of 15 was not successful in the use of a $10 \%$ Pd-C reagent, but the hydrogenation smoothly proceeded on a $20 \% \mathrm{Pd}(\mathrm{OH})_{2}-\mathrm{C}$ (Pearlman, 1967). The resulting hydroxyl group at 3'position was completly resistant to treatment with p-toluenesulfonic acid at room temperature. Treatments with $p$ toluenesulfonyl chloride/pyridine or pyridine-4-dimethylaminopyridine (DMAP) $/ \mathrm{CH}_{2} \mathrm{Cl}_{2}$ were also inactive and gave a mixture of 1-, 3'-tosylate and 1,3'-ditosylate in poor yields. Finally, 1-hydroxyl group of compound 15 was protected by silylation with tert-butyldimethylsilyl trifluoromethanesulfonate (TBDMSOTf), and the 1-silylate was successfully debenzylated with the Pearlman catalyst. Mesylation of 3'-position was achieved with a large excess of methanesulfonyl chloride/DMAP in $\mathrm{CH}_{2} \mathrm{Cl}_{2}$. The sulfonyl group of methanesulfonate was eliminated by treatment with 1,8-diazabicyclo[5.4.0]undec-7-ene (DBIJ) in refluxing toluene to furnish $1 \alpha$-tert-butyldimethylsilyloxy-6 $\alpha$-(2,2-dimethyl-7methoxychromen-6-yl)-2-oxo-3,7-dioxabicyclo[3.3.0]octane (18) in an acceptable yield. Thus, precocene framework in a haedoxan structure was constructed from 6 through 13 steps in a $9.2 \%$ overall yield. The tert-butyldimethylsilyl function was deprotected by tetrabutylammonium fluoride (TBAF) in THF, the resulting $6 \alpha$-(2,2-dimethyl-7-methoxychromen-6-yl)-1 a-hydroxy-2-oxo-3,7-dioxabicyclo[3.3.0]octane (19) was converted to 1 $\alpha, 2 \alpha / \beta$-dihydroxy-6 $\alpha$-(2,2-dimethyl-7-methoxychromen-6-yl)-3,7-dioxabicyclo[3.3.0]octanes (20) by diisobutyl aluminumhydride (DIBAL) reduction. The lactol was reacted with 2,6-dimethoxyphenol in the presence of a catalytic amount of pyridinium $p$ toluenesulfonate (Takano et al., 1988) in dry benzene under reflux to furnish the target compound $2 \alpha$-(2,6-dimethoxyphenoxy)-6 $\alpha$-(2,2-dimethyl-7-methoxychromen-6-yl)-1 $\alpha$ hydroxy-3,7-dioxabicyclo[3.3.0]octane (21). The stereochemistry was assigned from the chemical shift values by comparison with those of the stereoisomers of phrymarolins and 
haedoxans. Thus, an insecticidal sesquilignan analogue possessing anti-juvenile hormone structure was synthesized from 3-benzyloxy-2,2-dimethyl-7-methoxychroman-6-carbaldehyde and $\beta$-vinyl- $\gamma$-butyrolactone through 16 steps in a $0.92 \%$ overall yield (Scheme 2,3 ).<smiles>C=CC(CO)C(CO)C(CO)c1cc2c(cc1OC)OC(C)(C)C(OCc1ccccc1)C2</smiles>

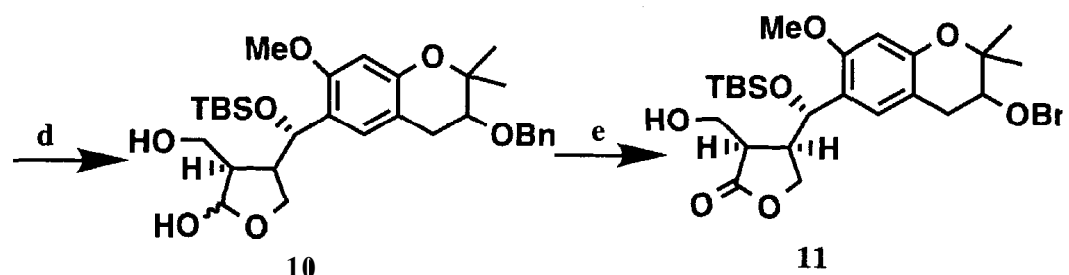

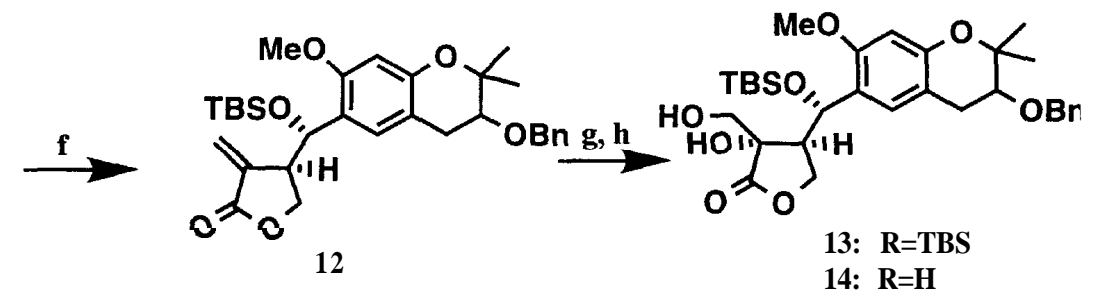

a) $\beta$-vinyl- $\gamma$-butyrolactone, KHMDS/THF; b) TBDMOTf, 2,6-lutidine/ $\mathrm{CH}_{2} \mathrm{Cl}_{2}$;

c) $\mathrm{LiAlH}_{4} / \mathrm{THF}$; d) (i) cat. $\mathrm{OsO}_{4}, \mathrm{NMO} / a q$. acetone, tert-BuOH, (ii) $\mathrm{NaIO}_{4} / \mathrm{H}_{2} \mathrm{O}$

e) $\mathrm{Ag}_{2} \mathrm{CO}_{3}$-celite/benzene; f) (i) $\mathrm{MsCl}, \mathrm{Et}_{3} \mathrm{~N} /$ benzene, (ii) $\mathrm{DBU} / \mathrm{benzene}$;

g) cat. $\mathrm{OsO}_{4}$, $\mathrm{NMO} / a q$. acetone, tert-BuOH; h) TBAF/THF

Scheme 2. Synthesis of the chromeno-lignan analogue.

\section{Bioassay}

The hybrid compound of insecticidal lignan/antijuvenile hormonal chromene was assessed to the 4th instar larvae of the silkworm (Bombyx mori), and this compound was inactive at a dose level of $200 \mu \mathrm{g}$ per larva. 


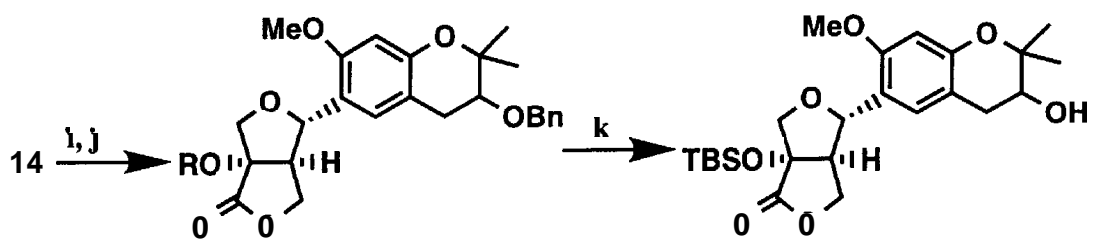

15: $\mathrm{R}=\mathrm{H}$

17

16: $R=T B S$<smiles>COc1cc2c(cc1[C@@H]1[C@@H]3OC[C@H](O)[C@@]3(O)CO[C@H]1c1cc3c(cc1OC)OC(C)(C)C=C3)OC(C)(C)C=C2</smiles>

18: $R=$ TBS

20

19: $\mathrm{R}=\mathrm{H}$

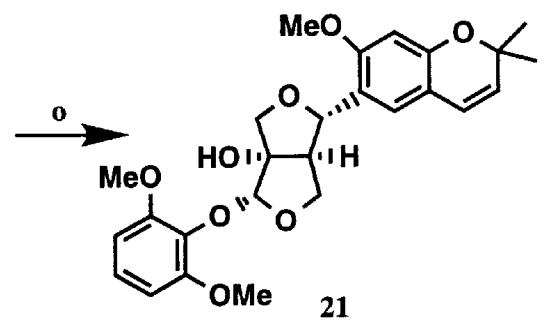

i) $\mathrm{CSA} / \mathrm{CH}_{2} \mathrm{Cl}_{2}$; j) TBDMSOTf, 2,6-lutidine/ $\left.\mathrm{CH}_{2} \mathrm{Cl}_{2} ; \mathrm{k}\right) \mathrm{H}_{2}, 20 \% \mathrm{Pd}(\mathrm{OH})_{2} / \mathrm{EtOAc}$;

I) (i) $\mathrm{MsCl}$, DMAP/CH$/ \mathrm{Cl}_{2}$; (ii) DBU/toluene; m) TBAF/THF; n) DIBAL/toluene

o) 2,6-dimethoxyphenol, cat. PPTS/benzene;

Scheme 3. Synthesis of the chromeno-lignan analogue (cont.).

\section{ACKNOWLEDGEMENTS}

We thank the staffs of the Center of Elemental Analysis, Faculty of Science, Kyushu University for the elemental analyses.

\section{REFERENCES}

Bowers, W. S. and T. Ohta, 1976 Discovery of insect antijuvenile hormones in plants. Science, 193: 542- 
Gotanda, H., S. Yamauchi, R. Takeya and E. Taniguchi, 1993 Insecticidal activity of lignan analogs: Chromano-sesquilignans with a dihydroprecocene skeleton. J.Fac. Agr., Kyushu Univ., 37: 349-358

Ishibashi, F. and E. Taniguchi, 1989 Syntheses of ( \pm )-Haedoxan A, D, E, and their stereoisomers. Agric. Biol. Chem., 53: 1565-1573

Ohta, T. and W. S. Bowers, 1977 Synthesis of insect antijuvenile hormones. Chem. Phrm. Bull., 25: 27882789

Pearlman, W. M., 1967 Noble metal hydroxides on carbon nonpyrophoric dry catalysts. Tetrahedron Lett., 17: 1663-1664

Takano, S., T. Ohkawa, S. Tamori, S. Satoh and K. Ogasawara, 1988 Enantiocontrolled route to the furofuran lignans: the total synthesis of (-)-sesamolin, (-)-sesamin and (-)-acuminatolide.J. Chem. Soc., Chem. Commun., 1988: 189-191

Taniguchi, E., K. Imamura, F. Ishibashi, T. Matsui and A. Nishio, 1989 Structure of the novel insecticidal sesquilignan, haedoxan A Agric. Biol.Chem., 53: 631-643

Yamauchi, S. and E. Taniguchi, 1991 Synthesis and insecticidal activity of lignan analogs (1). Agric. Biol. Chem., 55: 3075-3084

Yamauchi, S. and E. Taniguchi, 1992a Synthesis and insecticidal activity of lignan analogs (II). Biosci. Biotec. Biochem., 56: 412-417

Yamauchi, S. and E. Taniguchi, 1992b Synthesis and insecticidal activity of lignan analogs (III). Biosci. Biotec. Biochem., 56: 418-422

Yamauchi, S. and E. Taniguchi, 1992c Effect on insecticidal activity of substituents at the 1,4benzodioxanyl moiety of haedoxan. Biosci. Biotec. Biochem., 56: 1193-1197

Yamauchi, S. and E. Taniguchi, 1992d Synthesis and insecticidal activity of sesquilignan analogs with 2alkyl-6-methoxy-3-(3,4-methylenedioxyphenyl)-1,4-benzodioxanyl group. Biosci. Biotec. Biochem., 56: 1751-1759 